\title{
Estratégia de Migração de Serviço baseada em Informações Contextuais em uma Arquitetura MEC
}

\author{
Fábio Araújo $^{1}$, Derian Sobral ${ }^{1}$, Stefany Pinheiro $^{2}$, Helder Oliveira $^{1}$, Denis Rosário $^{1}$ \\ ${ }^{1}$ Universidade Federal do Pará (UFPA), Belém, Brazil \\ ${ }^{2}$ Centro Universitário do Pará (CESUPA), Belém, Brazil \\ fabioraraujoe.ufpa.br, derian.alencareitec.ufpa.br, \\ stefpinheirog@gmail.com, \{heldermay, denis\}@.ufpa.br
}

\begin{abstract}
Recently, mobile edge computing and service migration policies have shown promising results to improve the user experience and optimize the use of infrastructure resources. However, contextual information, in conjunction with the resources available on users' devices, has been ignored by most policies. In this paper, we propose a service migration strategy based on contextual information and evaluate the influence of user mobility on migration strategies. Simulation results highlight the superior performance of the proposed strategy compared to state-of-the-art algorithms and a performance equivalent to the optimal solution when the collection and analysis of context information is carried out properly.
\end{abstract}

Resumo. Recentemente, a computação móvel de borda e políticas de migração de serviço tem mostrado resultados promissores para melhorar a experiência dos usuários e otimizar o uso de recursos da infraestrutura. Entretanto, as informações contextuais, conjuntamente com os recursos disponíveis nos dispositivos dos usuários tem sido ignorados pela maioria das políticas. Neste trabalho, propomos uma estratégia de migração de serviço baseada em informações contextuais, definimos a solução ótima para os cenário, em termos de Qualidade de Experiência, e avaliamos a influência da mobilidade do usuário nas estratégias de migração. Os resultados obtidos destacam o desempenho superior da estratégia proposta comparada ao estado-da-arte e um desempenho equivalente à solução ótima quando a coleta e análise das informações de contexto são realizadas de forma adequada.

\section{Introdução}

De acordo com pesquisas recentes, os conteúdos multimídia representarão $82 \%$ do tráfego em 2022 [Forecast 2019]. Como uma tecnologia promissora para acomodar o crescimento explosivo de serviços móveis sensíveis a atrasos e com uso intensivo de computação, como avaliação de qualidade de vídeo, armazenamento em cache, balanceamento de carga, e outros, a computação móvel de borda (Mobile Edge Computing - MEC) tem atraído interesse de pesquisa de pesquisadores e engenheiros globais [Rosário et al. 2018]. A arquitetura MEC implanta funções de cálculo, armazenamento, processamento e outras funções na Estação Base (Base Station - BS) sem carregar tarefas para o data center em nuvem. A MEC tem uma série de benefícios, como redução do consumo de largura de banda, redução da latência ao localizar recursos em usuários próximos e maior eficiência de transmissão. No entanto, diminuir o atraso de transmissão e manter uma boa Qualidade de Experiência (QoE) é muito mais do que simplesmente implantar 
aplicativos e serviços móveis na extremidade da rede devido à mobilidade imprevisível do usuário e aos recursos disponíveis.

O usuário pode experimentar um grande atraso na resposta do serviço ou até mesmo uma interrupção do serviço se este estiver longe da BS onde o serviço correspondente é implantado. Para contornar estes problemas, uma política de migração de serviço eficiente e mecanismos de gestão de serviços são essenciais para decidir quando e para onde migrar o serviço de forma a garantir os requisitos de Qualidade de Serviço (QoS) e QoE na distribuição de conteúdos. Ao migrar proativamente serviços, tal como vídeo adaptativos, para nós de MEC, alguns pontos-chave são notados. 1) Recursos limitados: Armazenar uma quantidade alta de vídeos de taxa de bits podem ser desnecessários, mas caros, uma vez que os recursos de rádio disponíveis podem não ser suficientes; 2) Desperdício de recursos: Mais atenção deve ser dada ao custo do armazenamento em cache proativo, uma vez que várias versões do mesmo conteúdo de vídeo em termos de níveis de qualidade ou resoluções tem diferentes custos; 3) Qualidade e localização: Selecionar diferentes versões de taxa de bits e locais de armazenamentos para vídeos específicos afeta o nível de qualidade e o atraso de transmissão dos vídeos quando eles são buscados, afetando assim a QoE dos usuários móveis que assistem esses vídeos [Zhu et al. 2019].

Devido à grande variabilidade de nós e as vantagens e desvantagens intrínsecas das arquiteturas baseadas em MEC, o uso de informações contextuais apoia a tomada de decisão na escolha do local mais apropriado. A partir dessa perspectiva, este trabalho apresenta uma estratégia de migração de serviço baseada em informações de contexto em arquitetura MEC, denominada de Multiple-attribute-based Service Migration in Mobile Edge Computing networks (MSM-MEC). A política em arquitetura MEC permite coletar, receber e compartilhar informações contextuais (e.g., mobilidade do usuário, condições de rede, capacidade de armazenamento do nó, energia restante dos dispositivos e outros.) e armazena vídeos adaptativos para fornecer aprimoramento da QoE dos usuários, reduzindo o desperdício de recursos e descarregando o enlace de backhaul. Os resultados obtidos destacam o desempenho superior do algoritmo proposto em termos de desempenho de migração bem-sucedida e QoE dos usuários, em comparação com as soluções típicas e atuais e um desempenho equivalente a solução ótima quando a coleta e análise das informações de contexto são realizadas de forma adequada.

O restante deste trabalho está organizado da seguinte forma. A seção 2 descreve propostas existentes na literatura e suas principais diferenças. A seção 3 descreve a política de migração de serviço baseada em informações de contexto. A seção 4 discute a descrição e os resultados da simulação. A Seção 5 apresenta as conclusões.

\section{Trabalhos Relacionados}

Wang et. al. propôs uma estrutura de migração de serviço com reconhecimento de privacidade de localização eficiente para resolver o problema de preservação de privacidade de localização e migração de serviço simultaneamente [Wang et al. 2020]. Neste trabalho foi definido um custo total como a combinação do custo de migração, atraso percebido pelo usuário e o risco de perder a privacidade do local, e então formularam o processo de migração do serviço como um problema de processo de decisão markviano, com base na localização do usuário, com o objetivo de minimizar o total custo.

Quer et. al. propôs uma política de armazenamento proativa como uma possibilidade promissora para superar o paradigma reativo [Quer et al. 2018]. Eles forneceram uma expressão de forma fechada para o custo médio do sistema e derivaram uma estrutura de otimização para o serviço de armazenamento. Os resultados mostram o desempenho 
das políticas ótimas e heurísticas para uma política estática (não proativa), mostrando que pode ter uma redução muito significativa do custo do sistema para todos os níveis de mobilidade do usuário quando os usuários compartilham um interesse comum em alguns arquivos.

Outro fator importante considerado pelo estado da arte é o gerenciamento de recursos para evitar a escassez de recursos limitados, como armazenamento, energia e largura de banda da rede. Desta forma, Vo et. al. propôs um armazenamento em multicamadas e solução de compartilhamento de recursos que permite aos usuários móveis receberem versões de vídeo através de comunicações realizadas entre dispositivos, Estações-base Femto e Estações-base Macro em redes 5G ultra densas [Vo et al. 2020]. Em particular, este trabalho considera recursos de downlink e recursos de armazenamento em células macro, femto e nós móveis, a fim de satisfazer o usuário móvel que solicita um vídeo específico.

Apesar dos trabalhos relacionados desenvolverem estratégias para redução do custo do sistema, melhorar QoE, diminuir latência, nenhum dos estudos mencionados desenvolveram uma estratégia que considera a energia, mobilidade, armazenamento e vazão, de forma conjunta, como requisitos para migração de serviço em dispositivos móveis.

\section{Estratégia de Migração de Serviços em Arquiteturas MEC}

Nesta seção, é apresentado a estratégia de migração de serviço, denominada de MSMMEC. A estratégia considera a energia restante e mobilidade dos usuários, bem como as condições da rede como informações contextuais para tomada de decisão de migração.

\subsection{Modelagem da Rede e do Sistema}

No cenário específico, são implantados um conjunto de micro BS $b \in \mathcal{B} \equiv\left\{b_{1}, \ldots, b_{S B S}\right\}$ e macro BSs $m \in \mathcal{M} \equiv\left\{m_{1}, \ldots, m_{M B S}\right\}$. Assumiu-se que as áreas de cobertura das micro BSs não se sobrepõem, portanto, um usuário não pode ser conectado a várias micro BSs simultaneamente. Cada usuário $u \in \mathcal{U} \equiv\left\{u_{1}, \ldots, u_{U}\right\}$ é móvel e está sempre conectado a uma macro BS $m$ ou o micro BS $b$ mais próximo. Um conjunto de usuários conectados na mesma micro BS $b$ é denotado como $\mathcal{U}^{b}$.

O tempo foi dividido em intervalos de tempo que são rotulados com um índice discreto $t \in \mathbb{N}$. Em cada intervalo de tempo, os usuários podem solicitar um arquivo e o servidor migra proativamente os serviços para uma área específica (nós móveis, microcélulas ou macro-células). O arquivo solicitado pode ser transmitido através de um enlace $\mathrm{D} 2 \mathrm{D}$, se o arquivo estiver disponível no cache local de um dos outros usuários da mesma área, caso contrário, é entregue através de um enlace LTE pela micro ou macro BS.

O princípio fundamental do MSM-MEC é garantir que os requisitos para migração de serviço sejam atendidos e os problemas de atrasos sejam mitigados. Desta forma, é possível fornecer a melhor QoE ao usuário final, nas condições desse contexto. Isso significa que a qualidade e a localização do vídeo podem ser limitadas devido fatores de contexto, como mobilidade do usuário, capacidade de armazenamento do nó ou energia disponível.

Os arquivos são classificados de acordo com a popularidade, e cada usuário irá solicitar aleatoriamente um arquivo $f \in \mathcal{F}=\{1,2, \ldots, F\}$ de uma biblioteca de conteúdo de tamanho $F$. Desta forma, a popularidade de um conteúdo solicitado por um usuário segue uma distribuição Zipf com o parâmetro $\alpha$, conforme mostrado na Equação 1. 


$$
\mathrm{Z}_{u}^{f}=\frac{i_{u}(f)^{-\theta}}{\sum_{j=1}^{\mathcal{F}} j^{-\theta}}
$$

Onde, $i_{u}(f)$ é a classificação do arquivo $f$ para o usuário $u$, e $\theta \geq 0$ descreve a distorção da popularidade do arquivo. Se $\theta=0$, todos os arquivos têm a mesma popularidade, enquanto no caso de valores altos de $\theta$, existem apenas alguns arquivos populares, enquanto os outros têm uma probabilidade muito baixa de serem solicitados.

Conforme ilustrado por [Chen et al. 2016], a mobilidade do usuário impacta o desempenho do cache e os recursos desperdiçados em redes celulares. É imprescindível levar em consideração a mobilidade do usuário e a distribuição dos nós para garantir uma alta qualidade do sistema e proporcionar uma migração de serviço sem desperdício de recursos. Contudo, alguns dos estudos existentes ignoram a mobilidade do usuário e tem sido comumente assumido que os usuários móveis estão sempre em um local fixo. Neste trabalho, foi definido $T_{u}^{n}$ como a probabilidade de um usuário $u$ estar no alcance de um nó n no momento $t+1$. É calculado com base na mobilidade do usuário e nas posições das células pequenas e macrocélulas.

\subsection{Qualidade do Sistema}

Mediante uma solicitação de migração de serviço pelo usuário $u$, diferentes ações podem ser tomadas dependendo de onde os recursos são alocados e, consequentemente, diferentes custos e recompensas são encontradas. No contexto de streaming de vídeo, os modelos hierárquicos geralmente armazenam conteúdo próximo ao usuário, devido aos benefícios como menor atraso de transmissão, possibilidade de uso de comunicações D2D e descarregamento de enlace de backhaul. Portanto, um arquivo solicitado $f$, que não está armazenado no cache local, é primeiro procurado nos dispositivos locais de usuários localizados na mesma área, i.e., $\mathcal{U}^{b}$. Se falhar, a presença do arquivo $f$ é verificada em $\mathcal{B}$ e, em seguida, em $\mathcal{M}$. Finalmente, se todas as buscas anteriores falharem, o arquivo $f$ é solicitado ao servidor, que contém todos os arquivos da lista $\mathcal{F}$.

Com base nisso, atribuímos requisitos de rede, armazenamento e energia para cada nó $n \in \mathcal{N}=\{\mathcal{B} \cup \mathcal{U} \cup \mathcal{M}\}$ que armazena um conteúdo ou serviço. Cada requisito é definido de acordo com o arquivo ou a migração de serviço solicitada. Por exemplo, supondo que um arquivo $f_{v}^{k}$ represente um vídeo $v$ com qualidade $k$ e $f_{v}^{w}$ represente o mesmo vídeo $v$ com qualidade $w$, se $k>w$ então, para armazenar o arquivo $f_{v}^{k}$ em um nó $n_{1}$ e transmitir de um usuário $u_{1}$, é necessária uma maior quantidade de armazenamento disponível e mais energia no nó $n_{1}$, e melhores condições de rede no link $l_{u_{1}}^{n_{1}}$ devido ao tamanho dos segmentos e do vídeo como um todo. Portanto, denotando $\tau_{f, u, n}^{n e t}, \tau_{f, n}^{S}$ e $\tau_{f, n}^{e n}$ como limites mínimos de rede, armazenamento e energia, respectivamente, e $N E T_{f, u, n}^{t+1}$, $S T_{f, n}^{t+1}$ e $E N_{f, n}^{t+1}$ valores instantâneos dessas características no momento $t+1$, definimos os seguintes requisitos:

$$
\begin{gathered}
N E T_{f, u, n}^{t+1} \geq \tau_{f, u, n}^{n e t}, \forall f \in \mathcal{F} ; \forall u \in \mathcal{U} ; \forall n \in \mathcal{N} \\
S T_{f, n}^{t+1} \geq \tau_{f, n}^{S}, \forall f \in \mathcal{F} ; \forall n \in \mathcal{N} \\
E N_{f, n}^{t+1} \geq \tau_{f, n}^{e n}, \forall f \in \mathcal{F} ; \forall n \in \mathcal{N}
\end{gathered}
$$


Considerando $\mathbb{1}\{W\}$ a função indicadora de um requisito $W$, denotamos com $\delta_{f, u, n}^{n e t}, \delta_{f, n}^{S}$ e $\delta_{f, n}^{e n}$ variáveis de estado binárias, fornecidas por:

$$
\begin{gathered}
\delta_{f, u, n}^{n e t}=\mathbb{1}\left\{N E T_{f, u, n}^{t+1} \geq \tau_{f, u, n}^{n e t}\right\}, \forall f \in \mathcal{F} ; \forall u \in \mathcal{U} ; \forall n \in \mathcal{N} \\
\delta_{f, n}^{S}=\mathbb{1}\left\{S T_{f, n}^{t+1} \geq \tau_{f, n}^{S}\right\}, \forall f \in \mathcal{F} ; \forall n \in \mathcal{N} \\
\delta_{f, n}^{e n}=\mathbb{1}\left\{E N_{f, n}^{t+1} \geq \tau_{f, n}^{e n}\right\}, \forall f \in \mathcal{F} ; \forall n \in \mathcal{N}
\end{gathered}
$$

$\delta_{f, u, n}^{n e t}, \delta_{f, n}^{S}$ e $\delta_{f, n}^{e n}$ representam o resultado de os requisitos atendidos para rede, armazenamento e energia, respectivamente. Eles são iguais a 1 se o requisito for atendido, e 0 caso contrário, i.e., $\delta_{f, u, n}^{n e t}, \delta_{f, n}^{S}$ e $\delta_{f, n}^{e n} \in\{0,1\}$. Desta forma, agora definimos como o produto de três variáveis binárias uma nova variável binária $\delta_{f, u, n}^{n e t, S, e n}$, determinada pela Equação 10 que também é $\in\{0,1\}$.

Em seguida, definimos $Q_{u}$ (como mostrado na Eq. 8) como a qualidade do usuário $u$ para todos $u \in \mathcal{U}, f \in \mathcal{F}$, e $n \in \mathcal{N}$, que está diretamente associado aos benefícios fornecidos e aos requisitos atendidos quando o nó armazena o conteúdo solicitado. Além disso, formalizamos a solução ótima da estratégia proposta, como solução de um problema de otimização pseudo-booleana (Eq. 9).

$$
\begin{aligned}
Q_{u}= & \sum_{f \in \mathcal{F}} \sum_{n \in \mathcal{N}}\left(\frac{\alpha}{d_{u}^{n}}+\beta S_{n}\right) T_{u}^{n} Z_{u}^{f} \delta_{f, u, n}^{n e t, S, e n} \gamma_{u}^{n} \\
& \underset{\gamma_{u}^{n}}{\operatorname{maximize}} Q=\sum_{u \in \mathcal{U}} Q_{u}
\end{aligned}
$$

subject to

$$
\begin{gathered}
\sum_{n \in \mathcal{N}} \gamma_{u}^{n}=1, \forall u \in \mathcal{U} \\
\delta_{f, u, n}^{n e t, S, e n} \in\{0,1\} \\
\delta_{f, u, n}^{n e t, S, e n}=\delta_{f, u, n}^{n e t} \delta_{f, n}^{S} \delta_{f, n}^{e n}
\end{gathered}
$$

Onde assumimos que $d_{u}^{n}$ é o atraso do link do nó $u$ para o nó $n$, e $S_{n}$ o armazenamento disponível no nó $n$. Além disso, $\alpha$ e $\beta$ são constantes de normalização e que pesam a importância de $d_{u}^{n}$ e $S_{n}$ na qualidade do sistema, devido aos diferentes valores e unidades de cada um.

A resolução do problema de otimização pseudo-booelano mostrado anteriormente resulta na solução ótima para um cenário de migração de serviços em arquitetura MEC, ciente de informações de contexto, porém de alta complexidade. Baseado na formulação do problema, propomos uma política de migração de serviços em arquitetura MEC que coleta e utiliza as informações de contexto para tomada de decisões, nas quais resultam no melhor local para migração do serviço solicitado.

O desempenho da política de migração de serviços depende diretamente da forma com que as informações são utilizadas e coletadas. Para o cenário proposto, utilizamos 
a média móvel ponderada, como descrito em [Coelho et al. 2017], para obter valores de predição da vazão disponível no momento $t+1$. Da mesma forma, a fim de analisar a influência nas políticas de migração de serviço, utilizamos soluções de predição de mobilidade do usuário com diferentes acurácias.

A política MSM-MEC recebe os valores preditivos e as informações dos dispositivos a cada nova requisição de migração de serviço. Especificamente neste cenário, onde é realizada a migração de vídeos adaptativos, as informações são transmitidas a cada reprodução de um conjunto de segmentos de vídeo. Além disso, a política incorpora algoritmos proativos de adaptação de vídeos como ESBA [Araújo et al. 2019], responsável pela seleção da qualidade do segmento que deve ser armazenada.

Uma vez obtida as informações, a MSM-MEC define, através dos requisitos mínimos estipulados nas Eq. 2, Eq. 3 e Eq. 4 o conjunto de possíveis soluções para o problema. Depois de definido o conjunto, a MSM-MEC prioriza nós com menor atraso médio nas últimas transmissões e posteriormente aqueles que tem maior espaço de armazenamento disponível. Para otimizar o desempenho da estratégia proposta, os nós que não atendem a um dos requisitos mínimos necessários para a transmissão do vídeo solicitado, são descartados do conjunto de possíveis soluções sem a necessidade de verificar os demais requisitos.

\section{Metodologia e Resultados}

Implementamos a estratégia proposta de migração de serviço e as demais estratégias para avaliação no simulador NS-3.30 ${ }^{1}$. NS-3.30 implementa a pilha de protocolo LTE para comunicação. Consideramos um cenário de arquitetura MEC denso de $1 \mathrm{~km} \times 1 \mathrm{~km}$ área com 4 macro-células cobrindo todo o cenário até certo ponto e 60 micro-células distribuídas pelo cenário.

As macro-células têm uma potência de transmissão de [46] dBm, enquanto as micro-células têm uma potência de transmissão de [23] dBm. A simulação considera o modelo de perda do caminho de Nakagami, que pode ser muito adequado para cenários urbanos e quatro vídeos diferentes codificados em sete qualidades (300 kbps, 500kbps, 1000 kbps, 2250 kbps, 4500 kbps, 9000 kbps e 13000kbps). Conduzimos 33 simulações com diferentes sementes geradas aleatoriamente. Os resultados mostram os valores com intervalo de confiança de $95 \%$. Para a resolução do problema de otimização pseudo-booleana, utilizamos o software IBM ILog CPLEX ${ }^{2}$ e os mesmos cenários foram utilizados para avaliação.

Nos resultados abaixo, MSM-MEC representa a proposta com $100 \%$ de acurácia na predição de mobilidade dos usuários. MSM-MEC70 e MSM-MEC50 representam a proposta com acurácias de $70 \%$ e $50 \%$, respectivamente. PMVCS, refere-se a política de migração denominada de "Proactive Multi-Bitrate Video Caching Strategy" por [Zhu et al. 2019] e Ótima refere-se a melhor solução (definida na sessão anterior), em termos de QoE, para os cenários avaliados.

A Figura 1 ilustra a a distribuição da alocação de serviços para diferentes políticas de migração. Na figura em questão nota-se uma distribuição aproximada entre MSMMEC e a solução Ótima. A diferença de $4 \%$ entre as duas abordagens deve-se a acurácia na predição de vazão da rede, que também tem influência direta na alocação dos serviços requisitados. Da mesma forma, a acurácia na mobilidade dos usuários também influencia

\footnotetext{
${ }^{1}$ http://www.nsnam.org/

${ }^{2}$ https://www.ibm.com/br-pt/analytics/cplex-optimizer
} 


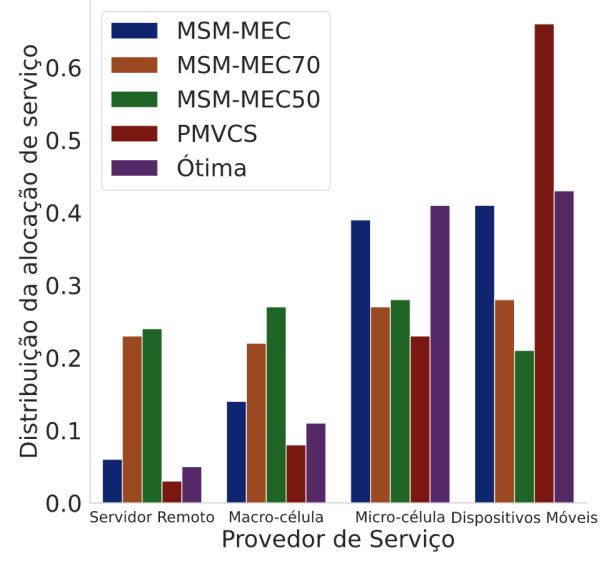

Figura 1. Distribuição da alocação de serviço.

no local em que os serviços serão disponibilizados. Enquanto MSM-MEC70 alocou 29\% dos serviços requisitados em locais diferentes da solução ótima, MSM-MEC50 alocou $36 \%$ dos serviços em outros locais.

A Figura 1 também ilustra a distribuição de serviços da política PMVCS que, apesar de destinar grande parte de seus serviços para dispositivos móveis próximos aos usuários responsáveis pelas requisições, ignora a energia dos dispositivos como um fator de decisão para alocação dos vídeos. Desta forma, parte da conexão entre os dispositivos são interrompidas e as requisições são realizadas novamente ao servidor, gerando atrasos e, possivelmente, perda na QoE do usuário como ilustrado na Figuras 2 e 3.

As Figuras 2 e 3 ilustram a taxa de bits média por usuário e a duração média de paradas por usuário, respectivamente. Observando a Figura 2, nota-se uma taxa de bits média maior utilizando a política de migração MSM-MEC, devido a acurácia no modelo de predição de mobilidade e análise das informações de contexto que colaboram para a tomada de decisão e diminuem o número de retransmissões dos vídeos solicitados. A medida que o número de usuários aumentam, a taxa de bits também aumenta, e a duração média de paradas do vídeo diminui, pois a maior quantidade de usuários permite um número maior de encontros entre os mesmos e possibilita destinar os vídeos solicitados para dispositivos próximos, desde que estes tenham os requisitos mínimos exigidos para migração.

\section{Conclusão}

Neste artigo apresentamos MSM-MEC, uma estratégia de migração de serviço capaz de escolher os melhores locais para armazenamento do vídeos solicitados. O MSM-MEC considera informações de contexto como mobilidade dos usuários, vazão disponível na rede, energia e armazenamento dos dispositivos para fornecer baixa latência e alta taxa de bits e baixo número de paradas dos vídeos solicitados. Com base nos resultados de simulação, mostramos que o MSM-MEC atinge um desempenho superior ao PMVCS e um desempenho próximo a solução ótima quando as predições são realizadas de forma adequada.

\section{Agradecimentos}

O presente trabalho foi realizado com apoio da Coordenação de Aperfeiçoamento de Pessoal de Nível Superior - Brasil (CAPES) - Código de Financiamento 001. 


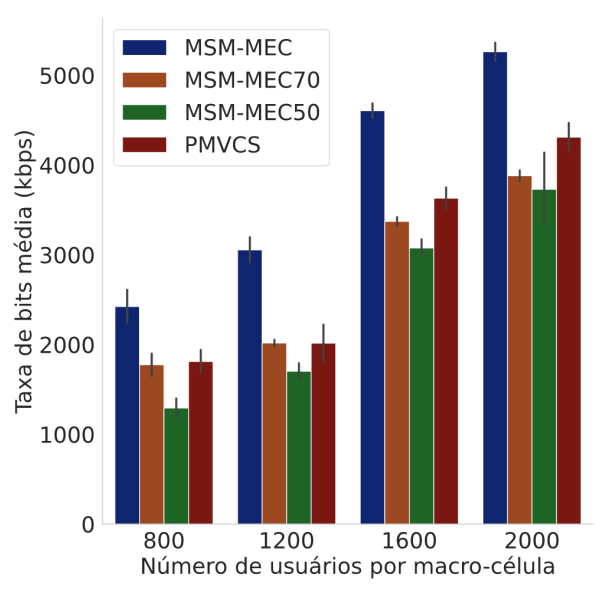

Figura 2. Taxa de bits média por usuário.

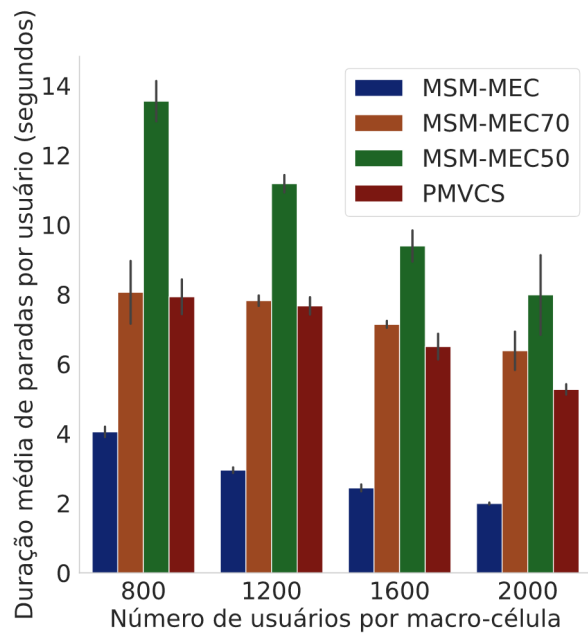

Figura 3. Tempo médio de paradas do vídeo por usuário.

\section{Referências}

Araújo, F., Rosário, D., Cerqueira, E., and Villas, L. A. (2019). A hybrid energy-aware video bitrate adaptation algorithm for mobile networks. In 2019 15th Annual Conference on Wireless On-demand Network Systems and Services (WONS), pages 146-153. IEEE.

Chen, M., Hao, Y., Qiu, M., Song, J., Wu, D., and Humar, I. (2016). Mobility-aware caching and computation offloading in $5 \mathrm{~g}$ ultra-dense cellular networks. Sensors, 16(7):974.

Coelho, M. d. S., Melo, C. A., and da Fonseca, N. L. (2017). Versioning-aware and qoe-oriented strategy for adaptative bitrate streaming. In 2017 IEEE International Conference on Communications (ICC), pages 1-6. IEEE.

Forecast, G. (2019). Cisco visual networking index: global mobile data traffic forecast update, 2017-2022. Update, 2017:2022.

Quer, G., Pappalardo, I., Rao, B. D., and Zorzi, M. (2018). Proactive caching strategies in heterogeneous networks with device-to-device communications. IEEE Transactions on Wireless Communications, 17(8):5270-5281.

Rosário, D., Schimuneck, M., Camargo, J., Nobre, J., Both, C., Rochol, J., and Gerla, M. (2018). Service migration from cloud to multi-tier fog nodes for multimedia dissemination with qoe support. Sensors, 18(2):329.

Vo, N.-S., Bui, M.-P., Truong, P. Q., Yin, C., and Masaracchia, A. (2020). Multi-tier Caching and Resource Sharing for Video Streaming in 5G Ultra-dense Networks. IEEE Communications Letters, 7798(c):1-1.

Wang, W., Ge, S., and Zhou, X. (2020). Location-Privacy-Aware Service Migration in Mobile Edge Computing. pages 1-6.

Zhu, H., Cao, Y., Hu, Q., Wang, W., Jiang, T., and Zhang, Q. (2019). Multi-Bitrate Video Caching for D2D-Enabled Cellular Networks. IEEE Multimedia, 26(1):10-20. 